\section{ANTIBODIES 1}

\section{Monday, July 6}

\section{OR.29. CD137 Deficiency Causes M Cell Defects and Low IgA Production}

En-Hui Hsieh, Xiomara Fernandez, Holly Eckelhoefer, David Lo UC Riverside, Riverside, $C A$

CD137 is a TNFR superfamily member known to contribute to $\mathrm{T}$ cell activation, but little is known about its role in mucosal immunity. We found that in CD137 deficient mice, Peyer's Patch UEA-1+ M cells showed an unusual narrow morphology with a surprising absence of basolateral pockets containing B lymphocytes. Similar morphological abnormalities were found in Nasal Associated Lymphoid Tissue (NALT) M cells. Functionally, NALT M cells in CD137 deficient mice also showed a marked inability to take up fluorescent microparticles. Since mucosal lymphoid tissues are associated with IgA isotype switching of $B$ cells, we asked whether the $M$ cell defects were associated with other immune defects. We found that CD137 deficient mice show poor fecal IgA responses to mucosal immunization, as well as very low total IgA levels. Surprisingly, their Peyer's Patch B cells had an unusually high percentage of IgA expressing B cells compared to wild type. These changes were specific to Peyer's Patch, as mesenteric and peripheral lymph nodes showed no abnormal increase in IgA + B cells. We are presently studying whether the defects are intrinsic to the hemopoietic cells or whether the defect is directly attributable to the observed M cell functional defect.

OR.30. Protective Monoclonal IgA Antibodies Have Direct Effects on the Virulence of Shigella Flexneri and Salmonella Typhimurium

Stephen Forbes ${ }^{2}$, Tia Bumpus ${ }^{1}$, Elizabeth McCarthy ${ }^{1}$, Nicholas Mantis ${ }^{1}$

${ }^{1}$ Wadsworth Center, Albany, NY; ${ }^{2}$ School of Public Health, University at Albany, Albany, NY

Secretory $\operatorname{IgA}(\mathrm{SIg} A)$ antibodies directed against the serotypespecific, O-antigens of the lipopolysaccharide (LPS) are the primary determinants of mucosal immunity to enteric bacterial pathogens, including Shigella flexneri and Salmonella enteric serovar Typhimurium. While the singular importance of SIgA in preventing enteric infections has been recognized for years, the underlying mechanisms by which this occurs remain largely unknown. IgAC5 is a serotype-specific, anti-LPS monoclonal IgA that has been shown to protect mice against experimental shigellosis. In this report, we demonstrate that IgAC5 is an effective inhibitor of $S$. flexneri's type three secretion system, the molecular apparatus on the surface of the bacterium involved in mediating uptake into intestinal epithelial cells. Secretion of the $S$. flexneri effector proteins IpaB and IpaC was reduced to virtually undetectable levels when the bacteria were exposed to IgAC5 $(5-10 \mu \mathrm{g} / \mathrm{ml})$ for as little as $15 \mathrm{~min}$. IgAC5 had no effect on bacterial viability, although antibody treatment did cause a reproducible reduction in the proton motive force across the bacterial inner membrane. These observations suggest that IgAC5, through its ability to associate with the O-antigen, may transiently compromise the integrity of the bacterial cell wall, and thereby affect both type three secretion and cellular bioenergetics. IgAC5 is not unique, as Sal4, a Salmonella O5 specific, anti-LPS monoclonal IgA, had similar effects on S. Typhimurium. These data represent the first demonstration that protective anti-LPS IgA antibodies may have direct effects on microbial virulence and reveal a previously unrecognized capacity of SIgA to potentially disarm enteric pathogens on mucosal surfaces.

\section{OR.31. Secretory Antibodies Affect Immune Homeostasis by Modulating Antigen Uptake}

Kim Simpfendorfer, Dimitri Diavatopoulos, Laura Del Mastro, Richard Strugnell, Odilia Wijburg

The University of Melbourne, Melbourne, VIC, Australia

Secretory antibodies (SAbs) are thought to play an important role in protection of the mucosal surfaces of our body against environmental antigens including pathogenic viruses and bacteria, normal flora and food. We used C57BL/6.pIgR knockout mice (B6.pIgR-/-) to demonstrate that the loss of SAbs results in increased susceptibility to infection by Salmonella typhimurium, as well as an increase in the translocation of antigen across the intestinal barrier. In this study we aimed to analyse the impact of the loss of SAbs on the immune homeostasis of the (gastrointestinal) immune system. Using histology and flow cytometry we have shown that there is an increase in central memory and activated T cells in Peyer's Patches and Mesenteric lymph nodes of B6.pIgR-/- mice but not in the spleen, and there is a greater number of lymphocytes into the gut lamina propria, presumably as the result of increased antigenic burden. Preliminary data suggests that there is increased uptake of $S$. typhimurium by dendritic cells and macrophages in the gut of B6.pIgR-/- mice, suggesting that the route of antigen uptake may be affected in the absence of SAbs. These findings have broad implications for the understanding of infection and antigen detection by the gut immune system.

OR.32. Breast Milk from HIV-infected Women in Uganda Inhibits HIV Infectivity with Neutralizing Immunoglobulin G

Jana Palaia ${ }^{1}$, Kristina Stoermer ${ }^{1}$, Laura Guay ${ }^{2}$, Michelle McConnell ${ }^{3}$, Mary Glenn Fowler ${ }^{2}$, Edward Janoff ${ }^{1}$

${ }^{1}$ University of Colorado Denver, Aurora, CO; ${ }^{2}$ Makerere UniversityJohns Hopkins University, Kampala, Uganda; ${ }^{3}$ Centers for Disease Control, Atlanta, GA

Post-natal transmission of HIV by breastfeeding occurs in $\approx 8-12 \%$, but not the majority, of HIV-exposed children in resource-limited countries. The functional HIV-inhibitory factors in breast milk are not well defined. To date, among milk samples diluted 1:4 from eight HIV-infected Ugandan women, six $(75 \%)$ showed greater than $50 \%$ inhibition of $\mathrm{p} 24$ production by a Ugandan clade A HIV strain (92UG031; 50 TCID $_{50} / \mathrm{mL}$ ) 
grown in PHA-activated PBMCs with IL-2. Neutralization was dose-dependent. No neutralizing sample was toxic to the target cells, and no antiretrovirals were detected. All milk samples showed HIV-specific IgG reactive with multiple HIV antigens, including the envelope binding and fusion domains (gp120 and gp41, respectively) by immunoblot, whereas IgA reacted only weakly with the core p24 antigen alone. Depletion of IgG from breast milk with Protein $\mathrm{G}$ microbeads diminished neutralization by $46-69 \%$ with concomitant elimination of HIV-specific IgG. These data demonstrate that breast milk from mothers with HIV can inhibit viral infection, that $\operatorname{IgG}$, not IgA, recognizes multiple epitopes of the virus and contributes substantially to viral neutralization. This activity may contribute to the selective rates of post-natal transmission of HIV to children in SubSaharan Africa. 